\title{
Experimental and computational study on epoxy resin reinforced with micro-sized OPEFB using rectangular waveguide and finite element method
}

\begin{abstract}
Epoxy resin (ER) composites reinforced with micro-sized oil palm empty fruit bunch (OPEFB) were fabricated to improve the biodegradability of electromagnetic interference connector gasket. The dielectric properties, transmission coefficient |S21|, reflection coefficient |S11|, reflection loss, power loss and shielding effectiveness were studied at a frequency range of 8-12 GHz. OPEFBER composites were prepared by varying the percentages of $\operatorname{OPEFB}(0,5,10,15,20,25,30$ and $40 \%$ ) at room temperature $\left(25^{\circ} \mathrm{C} \pm 2\right)$. Dielectric constant $\left(\varepsilon^{\prime}\right)$, loss factor $\left(\varepsilon^{\prime \prime}\right)$, reflection and transmission coefficients of the composites were measured using rectangular waveguide connected to vector network analyser. In addition, $\varepsilon^{\prime}$ and $\varepsilon^{\prime \prime}$ were used in finite element method technique to obtain $|\mathrm{S} 11|$ and $|\mathrm{S} 21|$. The results showed that the dielectric properties increased but $|\mathrm{S} 11|$ and $|\mathrm{S} 21|$ decreased with increasing OPEFB percentage in the composites. The shielding effectiveness, power loss and reflection loss increased with increasing OPEFB percentage in the composites. The simulated and measured results of $|\mathrm{S} 11|$ and $|\mathrm{S} 21|$ were in good agreement.
\end{abstract}

